\title{
SORO EM PÓ: ESTADO VÍTREO E CONDIÇÕES DE OPERAÇÃO DO SPRAY DRYER
}

\section{Whey powder: glassy state and spray drying conditions}

\author{
Ítalo Tuler Perrone ${ }^{I^{*}}$, Arlan Caldas Pereira Silveira ${ }^{2}$, Evandro Martins ${ }^{l}$, \\ Antônio Fernandes Carvalho ${ }^{I}$, Pierre Schuck ${ }^{3}$
}

\begin{abstract}
RESUMO
O conhecimento sobre a secagem de soro mudou significativamente nos últimos 20 anos. As indústrias começaram a utilizar em maior escala conhecimentos técnicos e científicos para a produção do soro em pó. Os principais problemas em soro em pó são: a adesão e o empedramento durante a fabricação e estocagem levando a diminuição do rendimento industrial. Estes problemas baseiam-se no controle do estado vítreo durante o processo de secagem e estocagem. Devido a importância da transição vítrea, os parâmetros de operação do spray dryer devem ser ajustados para melhorar o soro em pó. Esta revisão destaca os trabalhos científicos que abordam o impacto destes fatores sobre a secagem do soro. Como conclusão visando a melhoria do processo de secagem de soro recomenda-se que as indústrias: apliquem termohigrômetros para determinar as propriedades do ar, estabeçam a relação entre a atividade de água e a umidade relativa do ar de saída e ajustem os parâmetros de secagem baseados nas propriedades do ar e do soro.
\end{abstract}

Palavras-chave: transição vítrea; diagrama de Mollier; adesão; empedramento.

\begin{abstract}
The knowledge on spray drying of whey has significantly changed during the last 20 years. Industries started to apply more technical and scientific information for whey powder production. The main problems in whey powder are: sticking and

1 Universidade Federal de Viçosa (UFV), Av. Peter Henry Rolfs, s/n, Centro, 36570-900, Viçosa, MG, Brasil. E-mail: italotulerperrone@gmail.com

2 Gea Engenharia de Processos e Sistemas Industriais, Brasil.

3 UMR STLO, INRA,Agrocampus-Ouest, França.

* Autor para correspondência.
\end{abstract}

Recebido / Received: 04/10/2016

Aprovado / Approved: 25/01/2017 
caking during production and storage, which lead to decrease of the yield. These problems are based on the control of the glassy state during the dehydration process and storage. Due to the importance of the glass transition, spray drying parameters should be adjusted to improve whey powder production. This review highlights the scientific research that deal with the impact of these factors on spray drying of whey. To conclude, in order to improve the spray drying of whey the industries should: applythermohygrometers to measure the air properties; to stablish the relation between water activity and outlet air relative humidity; and to adjust spray drying parameters based on the properties of the air and whey.

Keywords: glass transition; Mollier diagram; stickiness; caking.

\section{INTRODUÇ̃̃O}

O conhecimento científico e tecnológico sobre a tecnologia de produtos lácteos concentrados e desidratados trata inevitavelmente dos efeitos da rápida remoção de água em um spray dryer sobre as configurações assumidas pelas moléculas presentes no alimento. As condições durante e após a secagem são propícias para o estabelecimento do estado vítreo. A manutenção do estado vítreo em lácteos desidratados é o mecanismo pelo qual alguns microrganismos (SCHUCK, 2014), compostos bioativos, proteínas e gorduras podem permanecer por longos períodos de tempo (6 meses a 2 anos) sem sofrerem modificações significativas em suas estruturas. Da mesma forma, o escurecimento e a ação enzimática também ficam minimizadas quando a matriz do produto desidratado conserva o estado vítreo. Alguns produtos, devido a sua composição, facilitam a manutenção do estado vítreo, conservando mais facilmente o produto e possibilitando um processo de secagem sem dificuldades (não ocorrendo adesão e nem empedramento do produto durante processamento e estocagem) (O'CALLAGHAN; HOGAN, 2013). O soro não é um produto no qual a manutenção do estado vítreo seja fácil, principalmente o soro ácido (ALVES et al., 2014; PERRONE et al., 2013; PERRONE et al., 2011).

\section{REFERENCIAL TEÓRICO}

O estado amorfo de um material se refere aos graus de liberdade de movimento de suas moléculas (estrutura molecular), em outras palavras, nenhuma posição exata das moléculas que constituem o material pode ser determinada. São exemplos de estados amorfos os estados líquido e gasoso dos materiais, enquanto que o estado cristalino é altamente organizado. O resfriamento de um líquido a uma temperatura inferior a sua temperatura de fusão, sem que ocorra a cristalização, retém a desordem molecular e as moléculas permanecem "estáticas" nas suas posições aleatórias, formando um tipo de sólido, porém desordenado (estado vítreo não cristalino).

A transformação sólido-líquido de um material amorfo é denominada transição vítrea. A transição vítrea é uma transformação reversível e ocorre em uma faixa de temperatura específica. Se as moléculas do sistema encontram-se a temperatura inferior a de transição vítrea, então estas estão "estáticas" nas suas posições originalmente aleatórias, com menores graus de liberdade para realizar rotações e vibrações, e, principalmente, com graus de liberdade limitados a translações, caracterizando o material como sendo tipo um sólido. Quando as moléculas do material encontram-se acima da temperatura de transição vítrea elas aumentam os graus de 
liberdade para a mobilidade translacional (super-resfriado), configurando um estado tipo um líquido, com concomitante aparecimento de um escoamento viscoso (ROOS, 2010, WOWK, 2010).

Sistemas no estado vítreo encontram-se fora do equilíbrio termodinâmico desta forma, caracteriza-se por ser um estado metaestável ou que simplificadamente possui a tendência de transitar para outros estados mais estáveis (como o estado cristalino) em uma magnitude de tempo que depende do material em questão e das condições de contorno (das condições das vizinhanças do material).

A secagem, devido a retirada rápida da água, propicia que moléculas que constituem o produto fiquem no estado vítreo. A formação deste estado vítreo nos produtos lácteos desidratados é o ponto chave para a conservação dos mesmos. O produto desidratado ser vítreo é importante para a conservação de alimentos, porque o estado natural desorganizado das moléculas da água e dos solutos presentes é preservado minimizando as perturbações do sistema, conservando-o
(WOWK, 2010). Durante a secagem, a lactose vítrea é formada a partir da lactose presente na solução do soro concentrado atomizado, uma vez que a retirada da água acarreta um rápido aumento na viscosidade, impossibilitando a cristalização. As técnicas de spray drying e de freeze drying são tipicamente empregadas para a produção de material vítreo (CARPIN et al., 2016).

Segundo Roos (2010) o sucesso da técnica de spray drying na estabilização de alimentos reside no controle do estado vítreo durante a secagem e estocagem dos produtos. Este ponto é determinante, pois apesar do estado vítreo ser desejado para a obtenção de produtos lácteos desidratados pelas vantagens de conservação do mesmo, deve-se ressaltar que este estado é "transitório" (metaestável). Ou seja, dependendo da composição do produto a ser seco, das condições operacionais aplicadas no spray dryer e das condições de transporte e armazenamento (umidade relativa do ar e temperatura) dos produtos desidratados os mesmos podem não permanecer no estado vítreo. Quando um produto lácteo possui

\section{Mudança de estado e transição vítrea}

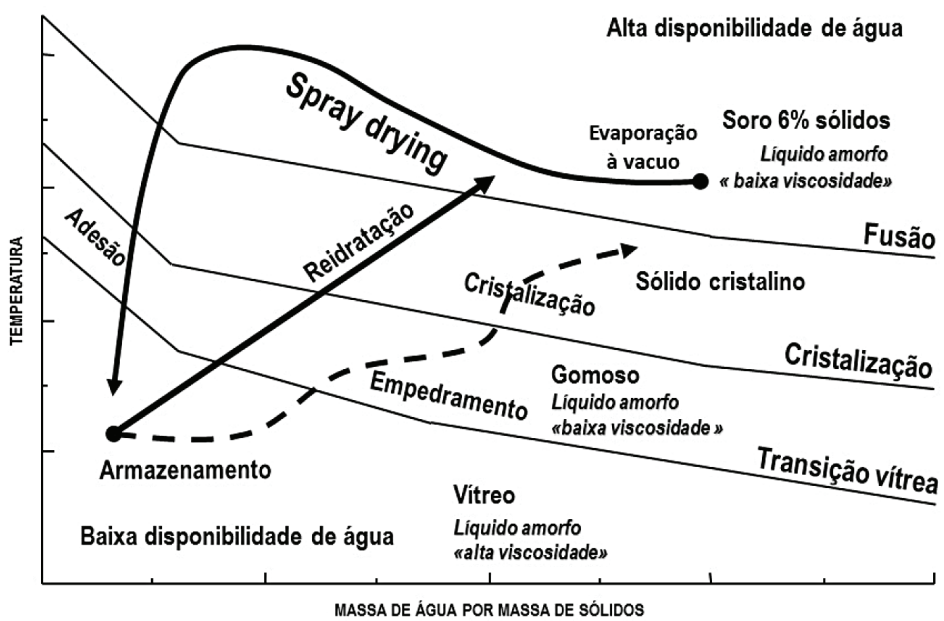

Fonte: Schuck (2015).

Figura 1 - Representação das mudanças de estado durante a produção de soro em pó 
facilidade para permanecer nas condições de processamento, transporte e estocagem no estado vítreo define-se como sendo um produto de fácil secagem (elevada dryability), caso contrário o produto é de difícil secagem (baixa dryability). São exemplos de produtos fáceis de secar (aqueles nos quais o estado vítreo permanece por mais tempo sob diferentes condições de processamento, transporte e estocagem) o leite em pó integral, os concentrados de proteínas do leite e do soro. São exemplos de produtos difíceis de secar o soro, o permeado de ultrafiltração e o leite com lactose hidrolisada.

A Figura 1 esquematiza o processo de fabricação de soro em pó por meio da definição do estado termodinâmico das moléculas do produto, em função da temperatura e do teor de umidade dos mesmos.

O soro com $6 \%$ de sólidos encontrase na região do gráfico na qual os graus de liberdade para movimentação das moléculas é grande e define-se que se encontra como um líquido amorfo de baixa viscosidade (amplos graus de liberdade para os movimentos transacionais, rotacionais e vibracionais de suas moléculas). Na sequência da fabricação do soro em pó, o produto é concentrado em evaporador a vácuo e inicia-se o processo de secagem. Durante a secagem a taxa de retirada de água é rápida e o aumento na viscosidade intenso, fazendo com que o produto atinja o estado vítreo desejado.

Se durante a secagem os produtos atingirem uma temperatura superior a temperatura de transição vítrea ( $\mathrm{Tg}$ ), dois fenômenos podem acontecer em sequência: a adesão e o empedramento. A adesão pode ser definida como a formação de pontes líquidas contendo açúcares amorfos ou lípides ou outro material dissolvido tendo como resultado elevada tensão interfacial entre as partículas do pó e as superfícies do equipamento. $\mathrm{Na}$ prática observa-se o início da formação de depósitos de produto sobre as superfícies dos equipamentos (spray dryer, fluidizador, tubulações e ciclones). Em sequência ao fenômeno da adesão pode-se observar, principalmente na estocagem, o fenômeno do empedramento.

O empedramento pode ser definido como a formação de aglomerados friáveis ou duros com diferentes tamanhos durante a produção, o transporte e a estocagem dos pós (CARPIN et al., 2016). A consequência é a formação de "blocos" ou "pedras" de produtos desidratados. Estes processos caminham para que o produto atinja um estado termodinâmico mais estável, o estado cristalino, sendo um processo exotérmico e indesejado durante a produção do soro em pó. De acordo com Palzer (2005) durante a transição vítrea a viscosidade do estado vítreo, de magnitude ao redor de $10^{12}$ Pa.s, diminui para um valor entre $10^{8} \mathrm{~Pa}$.s e $10^{9} \mathrm{~Pa} . \mathrm{s}$, e, desta forma, as moléculas no estado vítreo amorfo passam inicialmente para um estado gomoso e finalmente para um comportamento de plasticidade deformável.

Se durante a produção e o armazenamento as condições de contorno ao produto (principalmente umidade relativa do ar, temperatura e pressão mecânica) forem favoráveis, então o produto permanecerá no estado vítreo (líquido amorfo de alta viscosidade), conservando o alimento. Desta forma, devese evitar compactação mecânica, elevadas temperaturas de estocagem e ambientes com umidades relativas elevadas durante envase, transporte e estocagem do soro em pó.

A linha tracejada na Figura 1 representa um produto que durante a estocagem, devido ao contato com ar (elevada umidade relativa) e ou ambiente com temperatura superior a Tg, transitou do estado vítreo para o estado cristalino. Da mesma forma, temperaturas do ar de secagem muito elevadas na entrada e na saída do spray dryer também dificultam a manutenção do estado vítreo, levando à adesão e ao empedramento (cristalização do produto).

$\mathrm{O}$ conhecimento da $\mathrm{Tg}$ dos produtos pode ser uma importante ferramenta para 
o controle e para a produção de lácteos desidratados, em especial do soro em pó. $\mathrm{Na}$ Tabela 1 são apresentadas algumas temperaturas de transição vítrea para diferentes produtos.

Observa-se a partir da Tabela 1 que a temperatura de transição vítrea do leite em pó desnatado é de $92{ }^{\circ} \mathrm{C}$, e, em um primeiro momento, depreende-se que como o processo de secagem não propicia ao produto temperaturas próximas a este valor que os fenômenos de adesão e empedramento jamais ocorrerão na produção. Contudo, ao observarmos mais criteriosamente os dados apresentados na Tabela 1, constatamos que a Tg não é um valor absoluto, ou seja, ela varia de acordo com o teor de umidade do produto (ou da umidade do ar em equilíbrio com o produto). A temperatura de $92{ }^{\circ} \mathrm{C}$ para a transição vítrea é relativa ao leite em pó desnatado com teor de umidade que tende a zero. Se o leite em pó desnatado estiver em equilíbrio com o ar com $11,5 \%$ de umidade relativa, então apresentará umidade de $2,4 \%$ e Tg de $64{ }^{\circ} \mathrm{C}$. Da mesma forma se o equilíbrio ocorrer com o ar no qual a umidade relativa for de $33 \%$, então o teor de umidade do pó será de $7,1 \%$ e a $\mathrm{Tg}$ de apenas $33{ }^{\circ} \mathrm{C}$.

A água é o mais importante solvente, meio para dispersão e plasticizante em sistemas biológicos e alimentícios. Proteínas e carboidratos são os constituintes principais estruturantes dos alimentos e a interação destes com a água e entre eles na água governam a estrutura e as relações entre os constituintes em alimentos. A função entre a Tg e fração de volume da agua é chamada de função plasticizante (HELJO et al. 2012, MATVEEV et al. 2000). O comportamento que observa-se aqui consiste na capacidade das moléculas de água

Tabela 1 - Temperatura de transição vítrea $(\mathrm{Tg})$ para diferentes produtos

\begin{tabular}{l|c|c|c|c|c|c|c|c}
\hline \multicolumn{1}{c|}{ Produto } & $\begin{array}{c}\mathrm{Tg}\left({ }^{\circ} \mathrm{C}\right) \\
(\mathrm{UR} 0)\end{array}$ & $\mathrm{m}$ & $\begin{array}{c}\mathrm{Tg}\left({ }^{\circ} \mathrm{C}\right) \\
(\mathrm{UR} 1)\end{array}$ & $\mathrm{m}$ & $\begin{array}{c}\mathrm{Tg}\left({ }^{\circ} \mathrm{C}\right) \\
(\mathrm{UR} 2)\end{array}$ & $\mathrm{m}$ & $\begin{array}{c}\mathrm{Tg}\left({ }^{\circ} \mathrm{C}\right) \\
(\mathrm{UR} 3)\end{array}$ & $\mathrm{m}$ \\
\hline Lactose & 97 & 0 & 64 & 2,4 & 43 & 4,3 & 33 & 5,9 \\
Leite desnatado & 92 & 0 & 58 & 3,7 & 34 & 5,6 & 33 & 7,1 \\
Leite integral & 92 & 0 & 62 & 4,0 & - & - & - & - \\
Leite desnatado com & 49 & 0 & 26 & 1,5 & 5 & 3,0 & -8 & 5,2 \\
lactose hidrolisada & 31 & - & - & - & - & - & - & - \\
Glicose & 30 & - & - & - & - & - & - & - \\
Galactose & $62 \mathrm{a} \mathrm{70}$ & - & - & - & - & - & - & - \\
Sacarose & 132 & - & - & - & - & - & - & - \\
Caseína & 168 & - & - & - & - & - & - & - \\
Maltodextrina 6 DE & 130 & - & - & - & - & - & - & - \\
Maltodextrina 33 DE & 127 & - & - & - & - & - & - & - \\
Proteínas do soro & -139 & - & - & - & - & - & - & - \\
Água
\end{tabular}

Sendo: $\mathrm{Tg}=$ temperatura de transição vítrea; $\mathrm{UR}=$ umidade relativa do ar em contato com o produto a $24{ }^{\circ} \mathrm{C}$ após $24 \mathrm{~h}$; UR0 $=$ umidade relativa igual a $0 \%$; UR1 $=$ umidade relativa de $11,5 \%$, UR2 $=$ umidade relativa de 23,9\%; UR3 = umidade relativa de 33\%; $\mathrm{m}=$ massa de água $(\mathrm{g})$ por $100 \mathrm{~g}$ de sólidos dos produtos

Fonte: Hartel et al. 2011, Ergun, 2010, Schuck et al. 2005, Roos 2002, Kalichevsky 1993. 
de contribuírem para a diminuição da $\mathrm{Tg}$ dos produtos. Desta forma, produtos mais úmidos apresentam temperaturas de transição vítrea mais baixas do que produtos mais secos.

Segundo Schuck et al. (2005), a temperatura de transição vítrea de um produto lácteo pode ser entendida como a temperatura central média de todos os seus constituintes. A equação de Couchman-Karasz (1978) pode ser estendida a misturas ternárias de caseínas, carboidratos e água e é apresentada como a Equação 1.

$$
T \mathrm{~g}=\frac{\mathrm{w}_{1} \cdot \Delta \mathrm{Cp}_{1} \cdot T \mathrm{~g}_{1}+\mathrm{w}_{2} \cdot \Delta \mathrm{Cp}_{2} \cdot T \mathrm{~g}_{2}+\mathrm{w}_{3} \cdot \Delta \mathrm{Cp}_{3} \cdot T \mathrm{~g}_{3}}{\mathrm{w}_{1} \cdot \Delta \mathrm{Cp}_{1}+\mathrm{w}_{2} \cdot \Delta \mathrm{Cp}_{2}+\mathrm{w}_{3} \cdot \Delta \mathrm{Cp}_{3}}
$$

\section{Equação 1 - Equação Couchman-Karasz}

sendo: $T \mathrm{~g}=$ temperatura de transição vítrea (K); $T \mathrm{~g}_{1}, T \mathrm{~g}_{2}$ e $T \mathrm{~g}_{3}=$ temperaturas de transição vítrea dos componentes 1,2 e $3 ; \mathrm{w}_{1}, \mathrm{w}_{2}$ e $\mathrm{w}_{3}=$ as frações molares dos componentes 1,2 e 3 ; $\Delta \mathrm{Cp}_{1}, \Delta \mathrm{Cp}_{2}$ e $\Delta \mathrm{Cp}_{3}=$ variação da capacidade calorífera $\left(\mathrm{J} \cdot \mathrm{kg}^{-1} \cdot \mathrm{C}^{-1}\right)$ durante a transição vítrea dos componentes 1, 2 e 3; componentes 1, 2 e 3 = proteínas, carboidratos e água que compõem o produto lácteo.

A Equação 1 não leva em consideração frações que não estejam solubilizadas em água, como gordura, lactose cristalizada e fosfato de cálcio precipitado. Segundo Schuck et al. (2005), a variação da capacidade calorífera durante a transição vítrea dos componentes é representativa do grau de modificações bioquímicas que estão ocorrendo durante a transição. No caso da transição vítrea, que corresponde a uma mudança de um líquido amorfo com alta viscosidade para um líquido amorfo de baixa viscosidade, a variação da capacidade calorífera está diretamente relacionada com a magnitude das modificações que ocorrerão no produto durante a transição.
Compostos que possuem peso molecular mais elevado apresentam Tg mais elevada e desta forma contribuem para aumento da $\mathrm{Tg}$ do alimento. A relação entre peso molecular e Tg foi estabelecida para mais de 150 distintas moléculas presentes em alimentos nos trabalhos de Levine e Slade (1986, 1988a, 1988b, 1995). Desta forma, moléculas como a do ácido lático, da glicose, da galactose e da lactose tendem a diminuir a Tg do produto lácteo, ou seja, produtos cuja composição é rica em ácido lático, glicose, galactose e lactose tendem a apresentar Tg mais baixa e consequentemente a manutenção do estado vítreo nestes produtos é mais difícil, tornando o processo de secagem, transporte e estocagem destes produtos mais complicada. Um exemplo clássico deste comportamento é o soro ácido, composto rico em lactose e ácido lático, que apresentará baixa temperatura de transição vítrea e será de difícil secagem e conservação. Por outro lado, produtos cuja composição é rica em caseínas, proteínas do soro, fibras e maltodextrinas com baixo índice de dextrose equivalente (DE) apresentarão valores de Tg mais elevados e consequentemente serão mais fáceis de secar e conservar.

Pelo exposto acima, o controle da acidez em soro destinado a produção de soro em pó é determinante para o rendimento do processo, uma vez que soros com elevada acidez (intrínseca ao tipo de queijo produzido ou desenvolvida durante a estocagem e transporte do soro) serão de difícil secagem e conservação por apresentarem baixa $\mathrm{Tg}$ (dificuldade de manutenção do estado vítreo) (SILVEIRA et al, 2013).

Schuck et al. (2005) estudaram o efeito da diferença de temperatura entre a partícula do pó $\left(\mathrm{T}_{\mathrm{pp}}\right)$ e o valor da Tg do produto. Chegaram a conclusão que é possível prever o comportamento de diferentes tipos de pós durante a secagem (adesão) e estocagem (empedramento) por meio de dois atributos: 
$1^{\mathrm{o}}$ - a magnitude da diferença entre a temperatura da partícula do pó $\left(\mathrm{T}_{\mathrm{pp}}\right)$ e o valor da $\mathrm{Tg}$ do produto $\left(\mathrm{T}_{\mathrm{pp}}-\mathrm{Tg}\right) ; 2^{\mathrm{o}}-\mathrm{a}$ magnitude da variação da capacidade calorífera durante a transição vítrea. Desta forma, uma partícula de soro em pó submetida a temperaturas elevadas de secagem ou estocagem propiciará uma grande magnitude no valor de $\mathrm{T}_{\mathrm{pp}}-\mathrm{Tg}$. Nesta condição ocorrerá um aumento na velocidade das modificações físico-químicas do produto, como termoplasticidade, cristalização e reação de Maillard, levando consequentemente a adesão nos equipamentos, empedramento na embalagem e ao escurecimento acentuado do produto. No mesmo estudo os autores sugerem um índice de sensibilidade a adesão e ao empedramento denominado SCSI (stickiness and caking sensitivity index) que varia de $0 \mathrm{a}$ 10 e que pode ser aplicado a qualquer produto lácteo desidratado, com qualquer atividade de água e temperatura. O índice SCSI é calculado pela equação 2 .

$$
\begin{aligned}
\text { SCSI }= & \left(\text { número de pontos } \mathrm{T}_{\mathrm{pp}}-\mathrm{Tg}\right)+ \\
& (\text { número de pontos } \Delta \mathrm{Cp})
\end{aligned}
$$

\section{Equação 2 - Índice SCSI}

sendo: SCSI = índice de sensibilidade a adesão e ao empedramento, $\mathrm{T}_{\mathrm{pp}}-\mathrm{Tg}=$ diferença entre a temperatura da partícula do pós $\left(\mathrm{T}_{\mathrm{pp}}\right)$ e o valor da Tg do produto; $\Delta \mathrm{Cp}=$ magnitude da variação da capacidade calorífera durante a transição vítrea. Fonte: Schuck et al. (2005).

$\mathrm{Na}$ Tabela 2, segundo Schuck et al. (2005) pode-se determinar o número de pontos relativos para o cálculo do SCSI.

A manutenção do estado vítreo é determinante para a produção de lácteos desidratados e minimizar problemas de adesão e empedramento são objetivos industriais. A facilidade da manutenção do estado vítreo depende diretamente do tipo de moléculas presentes no produto (composição do produto), do teor de umidade final do produto e da temperatura da partícula do pó durante a secagem e estocagem (condições de operação do spray dryer e condições de transporte, estocagem e venda dos produtos).

\section{Condições operacionais do spray dryer}

A secagem dos líquidos por atomização ou pulverização, frequentemente chamada de secagem por atomização ou spray drying, consiste em dispersar o soro concentrado sob a forma de pequenas gotículas em uma corrente de ar quente, de maneira a obter soro em pó. Quando um alimento é colocado em uma corrente de ar com baixa umidade relativa (pressão de $1554 \mathrm{~Pa}$ ) e elevada temperatura

Tabela 2 - Cálculo do número de pontos para determinação do índice de sensibilidade a adesão e ao empedramento

\begin{tabular}{ccc}
\hline $\mathrm{T}_{\mathrm{pp}}-\mathrm{Tg}(\mathrm{oC})$ & $\Delta \mathrm{Cp}\left(\mathrm{J} \cdot \mathrm{kg}^{-1} \cdot \mathrm{C}^{-1}\right)$ & número de pontos \\
\hline$\left[\mathrm{T}_{\mathrm{pp}}-\mathrm{Tg}\right] \leq 5$ & $\Delta \mathrm{Cp}<0,1$ & 0 \\
$5<\left[\mathrm{T}_{\mathrm{pp}}-\mathrm{Tg}\right] \leq 10$ & $0,1 \leq \Delta \mathrm{Cp}<0,2$ & 1 \\
$10<\left[\mathrm{T}_{\mathrm{pp}}-\mathrm{Tg}\right] \leq 15$ & $0,2 \leq \Delta \mathrm{Cp}<0,3$ & 3 \\
$15<\left[\mathrm{T}_{\mathrm{pp}}-\mathrm{Tg}\right] \leq 20$ & $0,3 \leq \Delta \mathrm{Cp}<0,4$ & 4 \\
$20<\left[\mathrm{T}_{\mathrm{pp}}-\mathrm{Tg}\right] \leq 30$ & $0,4 \leq \Delta \mathrm{Cp}<0,5$ & 5 \\
$30<\left[\mathrm{T}_{\mathrm{pp}}-\mathrm{Tg}\right]$ & $0,5 \leq \Delta \mathrm{Cp}$ & 5 \\
\hline
\end{tabular}


(em média $200{ }^{\circ} \mathrm{C}$ ), é formada espontaneamente uma diferença de temperatura e pressão parcial de água entre o alimento e o ar, resultando uma transferência de energia na forma de calor do ar para o produto e uma transferência de água do produto para o ar, sendo que o ar em contato com a partícula láctea atomizada possui temperatura ao redor de $45^{\circ} \mathrm{C}$ e pressão de $9583 \mathrm{~Pa}$ (SCHUCK et al., 2010) (Figura 2). De acordo com Schuck (2012), as pequenas gotículas formadas e a grande área superficial das mesmas resultam em uma rápida evaporação da água a uma temperatura relativamente baixa, minimizando os danos térmicos ao produto.

Segundo Carić (2009) a taxa de variação infinitesimal da massa de água evaporada durante a secagem pelo tempo é expressa pelas equações 3 e 4 .

$$
\begin{aligned}
& \frac{\mathrm{dW}}{\mathrm{d} t}=\mathrm{A} \cdot \mathrm{K} \cdot(\mathrm{p} v k-\mathrm{p} v) \\
& \frac{\mathrm{dW}}{\mathrm{d} t}=\mathrm{A} \cdot h \cdot \frac{\left(\mathrm{T}_{v}-\mathrm{T}_{v k}\right)}{r}
\end{aligned}
$$

Equações 3 e 4 - Taxa de variação infinitesimal da massa de água evaporada sendo: $\mathrm{dW} / \mathrm{dt}=$ taxa de variação infinitesimal da massa de água evaporada durante a secagem pelo tempo $\left(\mathrm{kg} \cdot \mathrm{s}^{-1}\right) ; \mathrm{A}=$ área superficial da partícula atomizada $\left(\mathrm{m}^{2}\right)$; $\mathrm{K}=$ coeficiente de transferência de massa $\left(\mathrm{kg} \cdot \mathrm{s}^{-1} \cdot \mathrm{N}^{-1}\right) ; \mathrm{pvk}=$ pressão parcial da água na saturação na temperatura de bulbo úmido $(\mathrm{Pa})$; $\mathrm{pv}=$ pressão parcial da água no ar que envolve a partícula atomizada $(\mathrm{Pa}) ; \mathrm{h}=$ coeficiente de transferência de calor $\left(\mathrm{W} \cdot \mathrm{m}^{-2} \cdot \mathrm{K}\right) ; \mathrm{r}=$ calor latente de evaporação da água $\left(\mathrm{J} \cdot \mathrm{kg}^{-1}\right) ; \mathrm{Tv}$ $=$ temperatura do ar de secagem $(\mathrm{K}) ; \mathrm{Tvk}=$ temperatura de bulbo úmido $(\mathrm{K})$.

A velocidade de secagem é ligada a três fatores: a superfície de evaporação, a diferença da pressão parcial de água entre a gotícula e o ar e a velocidade de migração de água na gotícula (SCHUCK, 2012). De acordo com a lei de Fourier, quanto maior a área de troca, mais rápida será a transferência de energia na forma de calor e, portanto, maior será a velocidade de secagem. Acontece o mesmo para a cinética de secagem. Baseado na Equação 4, conclui-se que a transferência de água do produto para o ar é causada pela diferença da pressão parcial da água entre as gotículas e o ar seco e que, quanto maior a diferença, mais rápida será a secagem. Uma

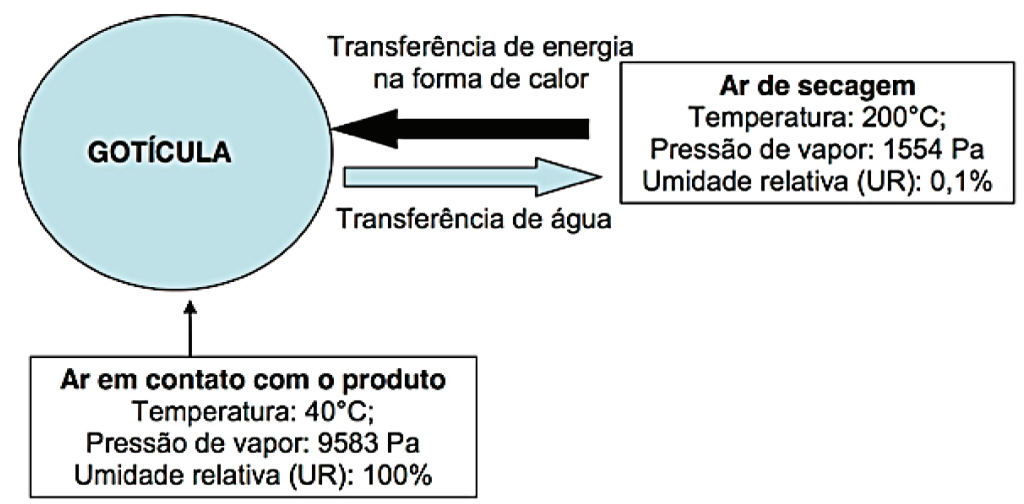

Fonte: Schuck et al. 2012.

Figura 2 - Esquematização do processo de secagem 
desidratação do ar e/ou um aumento da temperatura permite uma diminuição de sua pressão parcial da vapor.

Considerando o princípio apresentado, pode-se depreender que se o equilíbrio for alcançado entre o ar e as gotículas do soro, então ambos terão pressão de vapor idêntica. Ou seja, em condição de equilíbrio, a umidade relativa do ar (UR) que envolve as gotículas e/ou o produto em pó, possui magnitude igual ao valor da atividade de água do soro multiplicado por 100. Considera-se o equilíbrio entre o ar e as partículas, o momento no qual a taxa de transferência de água da partícula para o pó é exatamente igual a taxa de transferencia de água no pó para a partícula, desta forma, não há mudança significativa na composição do ar e nem do produto. Em termos industriais dificilmente o equilíbrio é alcançado, contudo é possível estabelecer uma relação entre a umidade relativa do ar de saída do spray dryer e a atividade de água do soro em pó para cada equipamento.

Schuck et al. (2005) estabeleceram a relação entre a umidade relativa do ar de saída da câmara com a atividade de água (Equações 5, 6 e 7) do soro em pó, leite integral e desnatado em pó (relações validadas experimentalmente para um spray dryer com três estágio para a secagem).

$$
\mathrm{UR}=40,2 \quad \mathrm{a}_{\mathrm{w}}-0,95
$$

Equação 5 - Soro cristalizado

$$
\mathrm{UR}=116,6 \quad \mathrm{a}_{\mathrm{w}}-16,38
$$

Equação 6 - Leite em pó integral

$$
\mathrm{UR}=105,6 \quad \mathrm{a}_{\mathrm{w}}-9,93
$$

Equação 7 - Leite em pó desnatado

sendo: $\mathrm{UR}=$ umidade relativa do ar de saída do spray dryer; $\mathrm{a}_{\mathrm{w}}=$ atividade de água do produto desidratado.

O estabelecimento das Equações 5, 6 e 7 nos equipamentos industriais de secagem possibilita o controle do processo de secagem do soro de forma mais eficiente. Classicamente os equipamentos são operados visando controlar a umidade final do soro em pó por meio da temperatura do ar de saída do spray dryer. Desta forma, se a umidade do soro em pó está acima do esperado então eleva-se a temperatura do ar de saída. A proposta de controle do spray dryer a partir do princípio de equilíbrio entre o ar e o produto consiste em não empregar a umidade do produto como o atributo principal de controle, mas sim empregar a atividade de água do produto seco. Desta forma, estipula-se o valor de atividade de água desejado no soro em pó (por exemplo $0,15)$ e controla-se as condições de secagem no spray dryer (vazão de soro concentrado no atomizador, massa de ar que entra da câmara, temperaturas do ar de entrada e de saída) por meio da determinação da umidade relativa do ar de saída da câmara (aplicando equações como as de número 5, 6 e 7). A partir deste tipo de controle é possível padronizar a atividade de água do soro em pó, atributo mais importante do ponto de vista da secabilidade e da conservação do produto do que o teor de umidade final. Para a realização deste tipo de controle é necessário o estabelecimento das equações que relacionam a umidade relativa do ar de saída da câmara com a atividade de água do equipamento específico e a instalação de termohigrômetros em pontos específicos do equipamento.

A utilização de termohigrômetros também possibilitará um controle do balanço de massa do processo de secagem do soro. Desta forma, por meio das Equações 8 e 9 é possível determinar a capacidade de evaporação do spray dryer. 


$$
\mathrm{AH}_{\mathrm{o}}=\mathrm{AH}_{\mathrm{i}}+\frac{\mathrm{m}_{\mathrm{a}}}{\mathrm{m}_{\mathrm{as}}}
$$

Equação 8 - Umidade absoluta do ar de saída

sendo: $\mathrm{AH}_{\mathrm{o}}=$ umidade absoluta do ar de saída do spray dryer $\left(\mathrm{kg} \mathrm{H}_{2} \mathrm{O} \mathrm{kg}{ }^{-1}\right.$ de ar seco); $\mathrm{AH}_{\mathrm{i}}$ = umidade absoluta do ar de entrada do spray dryer $\mathrm{m}_{\mathrm{a}}=$ vazão de água na alimentação do spray dryer via soro concentrado $\left(\mathrm{kg} \mathrm{h}^{-1}\right)$; mas = vazão de ar seco $\left(\mathrm{kg} \mathrm{h}^{-1}\right)$.

$$
\mathrm{m}_{\mathrm{a}}=\mathrm{m}_{\mathrm{c}}\left(1000-\mathrm{TS}_{\mathrm{c}}\right)
$$

Equação 9 - Vazão de água

sendo: $\mathrm{m}_{\mathrm{a}}=$ vazão de água do spray dryer via soro concentrado $\left(\mathrm{kg} \mathrm{h}^{-1}\right) ; \mathrm{m}_{\mathrm{c}}=$ vazão de soro concentrado na entrada do spray dryer $\left(\mathrm{kg} \mathrm{h}^{-1}\right) ; \mathrm{TS}_{\mathrm{c}}=$ massa de sólidos totais do soro concentrado $\left(\mathrm{g} \mathrm{kg}^{-1}\right)$.

Schuck et al. (2005) aplicaram o termohigrômetro como ferramenta para otimizar o processo de secagem, por meio da aplicação das equações de 5 a 9. Concluíram que quando a diferença entre o valor calculado para a umidade absoluta do ar de saída do spray dryer e o valor real medido durante o processo é menor do que $2 \mathrm{~g}$ de água por quilograma de ar seco, então nenhum problema de adesão no interior da câmara era observado. Contudo, quando a diferença é maior do $2 \mathrm{~g}$ de água por quilograma de ar seco, a adesão de produto no interior da câmara ocorre, correspondendo a uma remoção de água inferior a calculada, criando condições favoráveis a adesão. Desta forma, o operador pode acompanhar durante a secagem a variação da umidade absoluta do ar de saída do spray dryer e antecipar o problema da adesão por meio de correções nos parâmetros de secagem.

Variações na umidade absoluta e relativa do ar de saída do spray dryer (resultantes de flutuações na umidade absoluta do ar de entrada, no teor de sólidos do soro concentrado, na taxa de cristalização do soro ou na temperatura do ar de saída) podem ser rapidamente detectadas nas propriedades físicas do ar de saída da câmara ao se utilizar um termohigrômetro, evitando que estas flutuações afetem significativamente o teor de umidade, a atividade de água e a adesão do soro no equipamento.

Nos parágrafos anteriores fica evidente a importância de conhecer as propriedades físicas do ar durante a secagem de forma a se controlar o processo, evitar a adesão e possibilitar maior padronização do soro em pó obtido. A variação das propriedades do ar durante a secagem do soro podem ser representadas por meio do diagrama de Mollier (PÍSECKY, 1997) (Figura 3).

De acordo com Schuck et al. (2015), por meio da Figura 3 é possível acompanhar as propriedades do ar durante a secagem do soro. O ar ambiente que alimenta o spray dryer antes do aquecimento é representado pela letra $\mathrm{A}$. No ponto $\mathrm{A}$, o ar ambiente possui uma temperatura entre $20{ }^{\circ} \mathrm{C}$ e $30{ }^{\circ} \mathrm{C}$ e uma umidade absoluta do ar de aproximadamente $12 \mathrm{~g}$ de água por quilograma de ar seco. Ao passar pelo sistema de aquecimento de ar do spray dryer, o ar desloca-se para o ponto B do gráfico com temperatura de $180^{\circ} \mathrm{C}$. A partir deste ponto, o ar aquecido entra em contato com as gotículas de soro concentrado provenientes do atomizador que modificam. Observa-se uma mudança nas propriedades físicas do ar, após o contato com as gotículas de soro concentrado atomizadas, ficando este ar mais frio e mais úmido, diminuindo a sua temperatura e aumentando a sua umidade absoluta. Estas duas mudanças ocorrem simultaneamente e, considerando que toda a energia deste processo é transferida da forma de energia cinética das moléculas do ar para energia potencial das moléculas de água do soro, então o processo ocorre de forma 
isoentalpica. A consequência é o deslocamento das propriedades do ar do ponto B para o ponto $\mathrm{C}$.

Se considerarmos que no ponto $\mathrm{C}$ o ar de saída está em equilíbrio com o soro em pó, então definimos como sendo o momento final da secagem. No ponto $\mathrm{C}$, podemos observar que a temperatura do ar inicialmente em $180^{\circ} \mathrm{C}$ agora está próxima a $80^{\circ} \mathrm{C}$ (Tas), que é a temperatura do ar de saída da câmara. Ao prolongarmos o ponto $\mathrm{C}$ até a curva de $100 \%$ de umidade relativa do ar encontramos a temperatura de bulbo úmido (Tbu). Desta forma, a temperatura da partícula do soro durante a secagem localiza-se entre Tas e Tbu. A partir do ponto $\mathrm{C}$ também é possível observar a umidade absoluta do ar ao final da secagem (AHo) com valor aproximado de 52 de água por quilograma de ar seco. A diferença entre AHo e AHi (52 - $12=40 \mathrm{~g} \mathrm{H}_{2} \mathrm{O} \mathrm{kg}^{-1}$ de ar seco) indica a massa de água evaporada por quilograma de ar seco. Conhecendo-se a vazão de ar seco do equipamento é possível determinar a massa total de água evaporada durante a secagem do soro.

O Ponto C localiza-se sobre a curva correspondente a umidade relativa do ar de $20 \%$, desta forma, considerando o equilíbrio entre o ar de saída e a partícula é possível afirmar que nesta condição a atividade de água o soro em pó é de 0,200. Em condições industriais, equações como as equações 7,8 e 9 devem ser estabelecidas para determinar a correlação direta entre a umidade relativa do ar de saída da câmara com o valor da atividade de água do soro em pó.

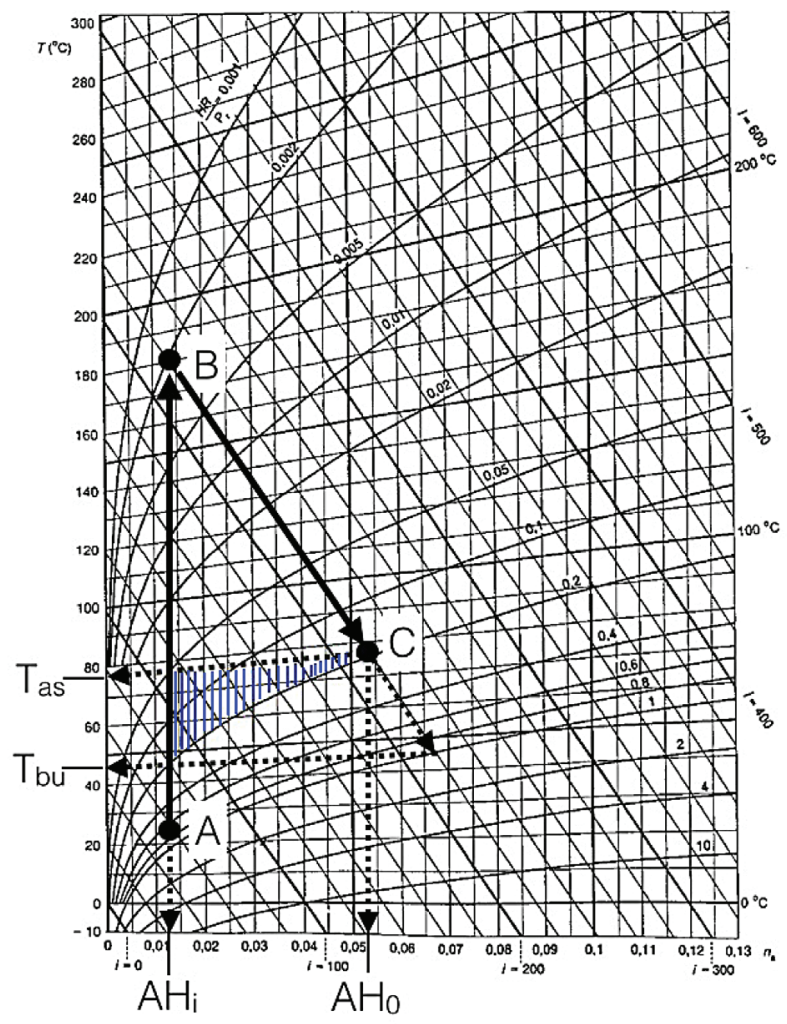

Figura 3 - Diagrama de Mollier aplicado a secagem do soro 
A região em azul no gráfico é a região definida como zona de segurança para a secagem de lácteos. Cada tipo de produto apresenta uma região no gráfico que não afeta de forma significativa a adesão do mesmo no equipamento. Deve-se destacar que a utilização da zona de segurança é determinante para a manutenção do estado vítreo, mas que podem exigir, dependendo do produto a ser seco, uma diminuição significativa da vazão de concentrado e das temperaturas de secagem, o que em conjunto levam a aumento nos custos operacionais e diminuição da capacidade produtiva. Nesta região observa-se que o soro em pó obtido estará sempre mais frio e mais seco, condições que favorecem o estado vítreo e consequentemente minimizam a adesão e o empedramento do produto.

Produtos de fácil secagem e estocagem apresentam zona de segurança ampla, indicando que pequenas alterações nos parâmetros de operação do spray dryer durante a secagem.

\section{CONSIDERAÇÕES FINAIS}

A manutenção do estado vítreo é o fundamento para a secagem do soro e deste conceito define-se quais os melhores parâmetros de operação devem ser utilizados em um spray dryer. A utilização de termohigrômetros, o estabelecimento da relação entre a atividade de água do produto e a umidade relativa do ar de saída da câmara e o emprego de diagramas de Mollier são as ferramentas tecnológicas principais para a secagem do soro de forma padronizada.

\section{AGRADECIMENTOS}

Os autores agradecem ao CNPq e a FAPEMIG pelo suporte financeiro a projetos na área de produtos lácteos concentrados e desidratados, e, à CAPES e ao CNPq pelas bolsas de pós-doutorado e de produtividade concedidas.

\section{REFERÊNCIAS}

ALVES, M. P. et al. Estudo da viscosidade de soluções proteicas através do analisador rápido de viscosidade (RVA). Revista do Instituto de Laticínios Cândido Tostes, v. 69, p. 77-88, 2014.

CARIĆ, M. et al. Technology of evaporators, membrane processing and dryers. In: TAMIME, A. Y. Dairy powders and concentrated products. Chichester: Wiley-Blackwell, 2009, p. 99-148.

CARPIN, M. et al. Caking of lactose: A critical review. Trends in Food Science \& Technology, v. 53, p. 1-12, 2016.

COUCHMAN, P. R.; KARASZ, F. E. A classical thermodynamic discussion of the effect of composition on glass transition temperatures. Macromolecules, v. 11, p. 117- 119, 1978.

ERGUN, R., LIETHA, R.; HARTEL, R. W. Moisture and Shelf Life in Sugar Confections. Critical Reviews in Food Science and Nutrition, v. 50, p. 162-192, 2010.

HARTEL, R. W.; ERGUN, R.; VOGEL, S. Phase/State Transitions of Confectionery Sweeteners: Thermodynamic and Kinetic Aspects. Comprehensive Reviews in Food Science and Food Safety, v. 10, p. 17-32, 2011.

HELJO, V. P. et al. The effect of water plasticization on the molecular mobility and crystallization tendency of amorphous disaccharides. Pharmaceutical research, v. 29, p. 2684-2697, 2012.

LEVINE, H.; SLADE, L. A polymer physicochemical approach to the study of commercial starch hydrolysis products (SHPs). Carbohydrate Polymers, v. 6, p. 213-244, 1986.

LEVINE, H.; SLADE, L. Principles of 
"cryostabilization" technology from structure/ property relationships of carbohydrate/water systems - a review. Cryo-letters, v. 9, p. 2163, 1988a.

LEVINE, H.; SLADE, L. Thermomechanical properties of small-carbohydrate-water glasses and 'rubbers'. Kinetically metastable systems at sub-zero temperatures. Journal of the Chemical Society, Faraday Transactions 1: Physical Chemistry in Condensed Phases, v. 84, p. 2619-2633, 1988b.

SLADE, L.; LEVINE, H. Water and the glass transition-dependence of the glass transition on composition and chemical structure: special implications for flour functionality in cookie baking. Journal of Food Engineering, v. 24, p. 431-509, 1995.

KALICHEVSKY M. T., BLANSHARD J. M. V., TOKARCZUK P. F., Effect of water content and sugars on the glass transition of casein and sodium caseinate, International Journal of Food Science and Technology, v. 28, p. 139-151, 1993.

MATVEEV, Y. I.; GRINBERG, V. Y.; TOLSTOGUZOV, V. B. The plasticizing effect of water on proteins, polysaccharides and their mixtures. Glassy state of biopolymers, food and seeds. Food Hydrocolloids, v. 14, p. 425-437, 2000.

O'CALlaghan, D. J., HOGAN, S. A. The physical nature of stickiness in the spray drying of dairy products: a review. Dairy Science \& Technology, v. 93, p. 331-346, 2013.

PERRONE, I. T.; PEREIRA, J. P. F.; CARVALHO, A. F. Aspectos tecnológicos da fabricação de soro em pó: uma revisão. Revista do Instituto de Laticínios Cândido Tostes, v. 66, n. $380,2011$.

PERRONE, I. T. et al. Isotermas de sorção e caracterização físico-química de lácteos desidratados. Revista do Instituto de Laticínios
Cândido Tostes, v. 68, n. 395, p. 33-38, 2013.

PÍSECKÝ, I. J.; Handbook of milk powder manufacture. Copenhagen: NIRO A/S, 1997, $261 p$.

ROOS, Y. H. Glass Transition Temperature and Its Relevance in Food Processing. Annual Reviews Food Science and Technology, v. 1, p. 469-96, 2010.

ROOS, Y. H. Importance of glass transition and water activity to spray drying and stability of dairy powders. Lait, v. 42, p. 475-484, 2002.

SCHUCK, P. Microbiology of Dried Milk Products. In: Batt, C. A.; Tortorello, M. L. Encyclopedia of Food Microbiology, Cambridge: Academic Press, 2014, p. 738-743.

SCHUCK, P. et al. Energy comsuption in the processing of dairy and feed powders by evaporation and drying. Drying technology, v. 33, p. 176-184, 2015.

SCHUCK, P. et al. Thermohygrometric sensor: a tool for optimizing the spray drying process. Innovative Food Science \& Emerging Technologies. v. 6, p. 45-50, 2005.

SCHUCK, P.; DOLIVET, A.; JEANTET, R. Analytical methods for food and dairy powders. Chichester: Wiley-Blackwell, 2012, p. 1-41.

SCHUCK, P. et al. Recent advances in spray drying relevant to dairy industry: A comprehensive critical review. Drying Technology, in press, 2016. <http://dx.doi.org/10.1080/07373 937.2016.1233114>.

SILVEIRA, A. C. et al. Spray drying: a review. Revista do Instituto de Laticínios Cândido Tostes, v. 68, p. 51-58, 2013.

WOWK, B. Thermodynamic aspects of vitrification. Cryobiology, v. 60, p. 11-22, 2010. 